\title{
Stress Recognition In Law Enforcement Organizations
}

\author{
Darrien R. Bush, Saint Leo University \\ Phillip Neely, Saint Leo University
}

\begin{abstract}
The study identified more critically the role of organizational stressors and impacts on police officer functions while on duty. Those aspects of stress-related responses were shown to be associated with agency managerial, administrative, and procedural responsibilities. Even so, while there appears to be some level of trepidation on a managerial level, the administrator has yet to effectively address problematic policies and procedures regarding stress recognition and supervisory accountability. In the study, the determinants of decision characteristics, perception characteristics, and motor act characteristics correlate with an officer's control of complex tasks (Violanti 1995). The focus of this study was to measure an officer's ability in stressful environments utilizing the Polar heart rate monitor during specific police activities. The highlighted areas of the study consisted of a physical agility test, firearm training, emergency vehicle operation, and voluntary exposure to taser deployment. The data collected were used for general observation into an officer's optimal performance in both low and high stress incidents.
\end{abstract}

Keywords: Trait Anxiety; Post Traumatic Stress Disorder (PTSD); Organizational Stress

\section{INTRODUCTION}

O nexpected shift changes for officers create a negative impact, as argued by Kohan and O'Conner (2002), which leads to extended work hours, rotating shifts, changes in days off, and a disruption in family responsibilities. The impact caused by abrupt, unsolicited organizational change can lead to displacement psychologically. Murtagh (2010) showed police officers have stress-based physical health problems, most notably high blood pressure, coronary heart disease, and gastrointestinal disorders. Waters and Ussery (2007) further showed that police officers suffer from respiratory dysfunction, eating disorders, insomnia, depression, and high rates of suicide. A stress-induced phenomena is directly related to and influenced by posttraumatic stress disorders (PTSD), as supported by the finding that "police officers, as an occupational category, are exposed to more acute and chronic life stressors than most other occupations and are vulnerable to the development of most, if not all, of these symptoms" (Waters and Ussery, 2007, p. 171).

Amplifying this finding, Collins \& Gibbs (2003) demonstrated the impact of anxiety (both state and trait) and task complexity on an officer's ability to respond to stress. The term state anxiety reflects an officer's present anxiety level in response to a particular stimulus whereas the term trait anxiety focuses on an officer's ability to function under multiple high-levels of anxiety. In the study, the determinants of decision characteristics, perception characteristics, and motor act characteristics correlate with an officer's control of complex tasks (Collins 2003). The focus of this study was to measure an officer's ability in stressful environments utilizing the Polar S810 heart rate monitor during specific police activities. The highlighted areas of the study consisted of a physical agility test, firearm training, emergency vehicle operation, and voluntary exposure to taser deployment. The data collected was used for general observation into an officer's optimal performance in both low and high stress incidents.

Stress in police officers' use of alcohol dependency was observed by Murtagh (2010) in a study on the Chicago Police Department. The study showed that $60 \%$ of police suicides were alcohol abuse related. Violanti (1995) argued that while difficulties are present in police suicide research, the past studies conducted in 1979 and 1990 equally showed an increased rate of alcohol-related suicides among police officers. From 1950 to 1979, on 
average there was one officer suicide every 2.5 years of the 2,662 officers sampled (Violanti, 1995). Additionally, from 1980 to 1990 this rate of police suicide had increased to one death every 1.25 years, which was an indication that police suicides rates had begun to double in frequency from previous studies.

The frequency in alcohol abuse suggests that police administrators should become aware of the workrelated problems associated with alcoholism (Greshon, Barocas, Canton \& Vlahov 2009; Violanti, 1995). Indicators cited by researchers are: increased vehicular traffic accidents, drinking on duty, absenteeism, and tardiness (Violanti, 1995). These indicators serve as managerial markers in identifying patterns of alcohol or drug dependency resulting from a larger problem in affected police officers. Violanti further argued that the increased percentage of officers who abuse alcohol also stems from the fear associated with nearing their retirement from policing.

Police officers' perception of retirement is determined by the longevity and distinction of their service. Most officers view retirement from the policing profession, as employees with any service organization do, as a positive occurrence of life's progression. However, the focus on separation from the policing subculture poses additional fears and anxieties due to officers' loss of status, self-definition, and friendships developed in that subculture.

Alcohol dependency can increase for officers who fear separation from the policing culture as they approach the age of retirement. Murtagh (2010) argued that as officers contemplate retirement from law enforcement, the use of alcohol becomes a coping mechanism for depression, anxiety, and the loss of camaraderie and security enjoyed with fellow officers. This bond established between officers is the result of years of service together that has isolated them from society and instilled an "us versus them" philosophy in relation to society in general. Ironically, as police officers retire from law enforcement, they are then ill-prepared to re-enter the very society they once perceived to be antagonistic, hostile, and a source of their stress.

\section{Organizational Stress}

Organizational stress affects police officers in a multitude of ways, such as excessive use of sick leave, tardiness, and requests for transfers. The consistency of agency leadership, disciplinary processes, and top-down communication facilitates this level of stress. Bennett and Hess (1996) argued that organizational stress also includes officers' workload, unfair treatment, mandatory overtime, limited opportunities for advancement, and insufficient pay. An officer's workload stress is precipitated by independent precinct assignment, work shifts, and the nature and frequency of priority calls. The effects of these contributors are manifested in the officer's psychological, physical, and emotional reactions to these stimuli. As Murtagh (2010) has shown, officers' responses to stress stimulants included, "increasing arrest rates, verbally and/or physically abusive, abusive to authority, abusing alcohol, decreasing caution, arguing, losing interest, and disobeying rules" (p. 25).

Organizational stress results from the paramilitary structure of policing agencies (Murtagh, 2010). Additionally, a rigid chain of command structure that utilizes the top-down communication approach, inconsistent disciplinary guidelines and practices, unstable policies and procedures, and discretionary promotional systems has been identified with contributing to organization stressors within policing agencies (Murtagh, 2010). Murtagh demonstrated the degree to which officers are subjected to organizational stress is also precipitated by their educational level. Murtagh found that police officers possessing advanced degrees were less likely to be impacted by organizational stress than were officers with high school diplomas. Asen and Colon (1995) showed that while police officers' adherence orders from superior officers is expected, officers possessing college degrees are more inclined to question decisions related to departmental policy and procedures.

Asen \& Colon (1995) studied a total of 271 police officers to determine the stressors with the greatest impact in policing. They found that organizational stress has the most impact on officers on duty, but that the highest levels of stress came from the death of a fellow officer. Violanti and Aaron (1993) surveyed the causes of external stress on police officers and determined that organizational stress was six times more likely to negatively impact police officers in the course of their duties. 
Collins and Gibbs (2003) studied organizational stressors to determine the mental health of police and law enforcement officers. They administered the General Health Questionnaire to 873 police personnel at the rank of sergeant and constable. The targeted items consisted of job satisfaction perception, assignment and shift work, life stressors, organizational operations, and buffers to stress. The study showed a high tendency towards organizational factors as the primary source of stress, even with minimal levels of operational contributors. The study further demonstrated that police officers exhibited a higher level of anxiety and nervousness, which contributed to general health concerns with lack of exercise, taking their work home, and exhibiting destructive behaviors to their peers while in public settings.

Collins and Gibbs (2003) noted a limitation in the General Health Questionnaire-a lack of detail in areas with low or high scores. This limitation requires further study to identify contributing factors not previously analyzed in the questionnaire design as related to routine assignments, unsolicited transfer orders, and discretionary promotions and appointments (e.g.. for detectives and training academy). McCarty, Zhao, and Garland (2007) later identified areas of discretionary assignments as a source of organizational stress, with officers' perceptions declining significantly towards merit promotions under the current system.

McCarty et al. (2007) described four stress-related contributors within the police organization:

1) Stress from the work environment,

2) Bureaucratic characteristics of police organizations,

3) Availability of peer support and trust, and

4) Accessibility of coping mechanisms. (p. 3)

The level of stress from the work environment was related to the daily situations of danger faced by police officers. The factors listed for stress contributors included death of a fellow officer, officer involved in fatal shooting, and the response to felony calls while in progress. Additional factors included the preservation and maintenance of a gruesome crime scene and evidence (p. 3). Volanti (1995) further demonstrated that police officers' high stress conditions are the primary contributors of their stress. The study also showed that officers under a constant barrage of stressors from police managers, the administration, and high priority calls daily often overwhelm officers at their strongest point. This vulnerability was shown to negatively impact officers' ability to cope with stress and often led to burnout.

The bureaucratic characteristic of policing organizations presents an additional organizational component to the stress endured by police officers. Volanti and Aaron (1993) defined this aspect of stress as "those events precipitated by the police administrations that are troubling or bothersome to members of the organization" (p. 4). Stressors ranged from lack of participation during policy development and vague or conflicting policy implementation to poorly trained first-line supervisors and inadequate disciplinary processes (Asen \& Colon, 1995). These stressors account for the increased stress levels in officers as their discretionary powers erode during the course of a tour of duty. Coman and Evans also demonstrated that the stress experienced by police officers furthered impacted negatively on female officers' anxiety and burnout rates.

\section{BACKGROUND}

Police administration can take proactive measures to address issues of organizational stress at the operational level. The availability of peer support and trust, according to McCarty, Zhao \& Garland (2007), would serve to mitigate circumstances of stress and burnout among police officers prior to a potential harmful encounter or experience. McCarty et al. (2007) showed that while police officers rely heavily on each other's support and trust, an organizational approach to support provides a higher degree of salience in law enforcement.

McCarty et al. (2007) further demonstrated the experience of interdependency among police officers. This connectedness affords officers the ability to understand and cope with the harsh realities of the profession (McCarty et al., 2007). Mazoni \& Eisner (2006) demonstrated through empirical research that within the policing culture, higher levels of internal support from their peers served as a mechanism to lower and control potential stressful situations encountered on the job. While Stinchcomb (2004) agreed with similar findings, additional findings 
suggested the lack of an internal support system within a police agency subjects female officer to higher levels of vulnerability than male officers. This finding relates to the high ratio of male officers to females along with male officers' propensity to demonstrate the politically stressful nature of police functions.

Police officers experiencing stressful situations are more inclined to forego available counseling offered by policing agencies. Police administrators are mandated to provide, at no cost to the employees, professional counseling to assist in traumatic situations both on and off duty. McCarty et al. (2007) noted that administrators must provide resources for positive coping mechanisms to ensure that officers respond to stress situations appropriately. Bennett \& Hess (1996) explained that this process is a cognitive or behavioral level strategy that reduces the negative effects of stress on officers, which in turn diminishes an officer's response to negative stimuli.

\section{LITERATURE REVIEW}

The literature review analyzed the nature of stress experienced on the job and its impending impact on decision making. As Collins \& Gibbs (2003) observed, African-American female police officers possess a unique perspective on issues deriving from their race and gender as this group has been largely ignored. This group has provided new knowledge to the current literature with a broader understanding of organizational patterns of racial sexism which will assist police administrator's development a more professional and diverse response appropriate to the needs of this specific subgroup.

Police officers experiencing organizational stress are prone to exhibit indicators of being burned out from the job. This concept of burnout refers to continuous negative impacts on an individual officer (Stinchcomb, 2004). During the study on organizational stressors, Stinchcomb demonstrated, "Similarly, the continuous grinding-away process eventually takes a toll on the work's physical, mental, and/or emotional health- a toll that may eventually appear as the psychological disorder known as 'burnout' (p. 262). This aspect of police work is more pronounced than in other service oriented organizations as police agency's operational capacities are performed in the public's view. This leads to increased interactions with the public, criminal element, and media scrutiny. Manzoni and Eisner (2006) described this form of burnout as, "Emotional exhaustion denoting the feeling of being emotionally depleted by contact with citizens. Depersonalization refers to negative, cynical attitudes and feelings toward citizens, leading to a dehumanized perception of citizens" (p. 11). Their study furthered demonstrated the internal implications for the officer during this stage of performance decline, leading to self-perceptions of inadequacy and loss of self-work.

As the literature review shows the link between stress and police use of excessive force, it becomes critically important to establish by empirically testing causal factors under the current study of poor supervision, lack of decision making input, inadequate support by departmental and supervisory personnel, and ineffective training in stress recognition.

\section{METHODOLOGY}

This quantitative study consisted of a survey administered to certified law enforcement personnel from policing agencies in the southeastern United States. Specifically, the Police Survey for Job Related Stress was utilized as the primary instrument of data collection. The study focused on the research questions and hypotheses:

- RHO1: There is no statistically significant difference between the impacts of organizational events on the use of excessive force as a factor of age for African American female police officers.

- $\quad \mathrm{RHO} 2$ : There is no statistically significant difference between the impacts of organizational events on the use of excessive force as a factor of years of experience for African American female police officers.

- $\quad$ RHO3: There is no statistically significant difference between the impacts of organizational events on the use of excessive force as a factor of rank for African American female police officers.

The questionnaire was administered during previous research and did not require a pilot test of its effectiveness as a survey tool. The survey focused specifically on the job-related impacts of stress as experienced by officers in the field. To clarify, in the field applied to those officers assigned to patrol functions, SWAT operations, and motorcycle unit traffic enforcement. 
The officers studied engaged in daily interactions with the public, citizen-police contact, and arrest or citation engagement during routine law enforcement procedures. The survey specifically measured how officers internalize the situations confronted daily during calls for service and help calls for officers in distress. Additionally, the survey considered how officers deal with the public's perception during routine procedures and their own potential skepticism towards the public (us versus them mentality).

\section{Data Collection and Analysis}

The selected design effectively measured and analyzed critical areas of police stress and anxiety levels during situations of alleged misconduct. This studys instrument was designed to extract data on officer's perception of stress to analyze trends, attitudes, and opinions relating to the use of force. This methodology afforded the most effective opportunity to gain both an objective and subjective view of the underlying factors contributing to the dynamics of the research question. Categorized in a descriptive statistical report the trends, opinions, and views held by the agencies' participants. The survey tool measured officers' perceptions in the areas of (a) confidence in police administrators, (b) organizational support and backing, (c) operational efficiency, and (d) trust in first-line supervision. In this regard, the primary aim of this research was to make known something previously unknown to police administrators, advance human knowledge, and prioritize the need for relevant and timely training. This approach allows police administrators to become proactive by implementing a training module designed for early detection of stress-induced behavioral changes in their sworn police officers.

An analysis of variance (ANOVA) was used to statistically compare the differences between the independent variable (organizational events) and the dependent variable (use of excessive force) for African American female police officers. The alpha level of 0.05 was set as the level of significance in determining whether to accept or reject the study's null hypotheses.

\section{CONCLUSIONS}

Police management will have at their disposal relevant data in which to analyze and measure its effectiveness on stress detection and early supervisor intervention. As identified by survey participants, there are unique stress contributors specific to the law enforcement environment. This study examined the presence and response to stress and its relationship to excessive use of force as perceived by African-American female police officers. Manzoni \& Eisner (2006) found the African-American female police officers will have varying experiences and perceptions within law enforcement from that of other highly studied sub-groups.

This study answered the research questions delineated at the outset of this study. The results revealed that there is no significant difference with respect to age, rank, and years of service for African-American female officers in their perception of stress and the use of excessive force within their respective agencies. These findings are critical to gaining new knowledge and adding to existing literature of the phenomenon associated with subgroups in law enforcement as police administrators develop training modules for a diverse law enforcement environment. The focus from this standpoint is directed toward a subgroup with a unique perspective due to the isolation associated with being African-American and female in the policing profession.

Police work is viewed as a paramilitary organization with rigidly structured policy and procedures. The research identified inconsistencies involved in these systems which were related to the organizational stress officers experienced, which became manifest in the use of excessive force. This highlights the need for proactive strategies at the management level to create effective mechanisms for early detection of stressors for supervisory intervention. As police work tends to fluctuate rapidly from a calm, controlled environment to one of high tension, unpredictability, and chaos, officers require adequate training in maintaining restraint to control an emotionallycharged climate.

In an environment of unpredictability, the proper training and two-way communication with line officers and management would create effective systems for early intervention thereby eliminating or reducing occurrences of excessive force resulting from unseen organizational stress factors. Police officers participating in the survey identified multiple stressors on the organizational level that requires additional reflection and review. Police 
administrators now possess the task of analyzing trends, data, and officer response to develop effective methods for restructuring policy in the areas of training, supervision, discipline, and program implementation.

\section{AUTHOR INFORMATION}

Dr. Darrien R. Bush is the recipient of the Doctor of Philosophy in Public Policy and Administration from Walden University and the Masters of Science in Public Administration from Columbus State University. He is an Associate Professor at Saint Leo University in Duluth, Georgia. Dr. Bush's expertise comes in the field of criminal justice and public policy.

Dr. Phillip Neely is the recipient of the Doctor of Philosophy in Public Policy and Administration from Walden University and the Masters of Science in Public Administration from Central Michigan University. $\mathrm{He}$ is an Associate Professor at Saint Leo University in Duluth, Georgia. Dr. Neely's expertise comes in the field of criminal justice and public policy.

\section{REFERENCES}

Asen, J., \& Colon, I. (1995). Acceptance and use of police department employee assistance programs. Employee Assistance Quarterly, 11, 45-54. doi.org/10.1300/J022v11n01_04

Bennett, W. W., \& Hess, K. M. (1996). Management and supervision in law enforcement (2nd ed.). St. Paul, MN: West Publishing Company.

Collins, P. A., \& Gibbs, A. C. C. (2003). Stress in police officers: A study of the origins, prevalence and severity of stress-related symptoms within a county police force. Occupational Medicine-Oxford, 53, 256-264. doi.org/10.1093/occmed/kqg061

Kohan, A., \& O'Connor, B. P. (2002). Police Officer Job Satisfaction in Relation to Mood, Well-being, and Alcohol Consumption. The Journal of Psychology, 136(3), 307-318. doi.org/10.1080/00223980209604158

Manzoni, P. \& Eisner, M. (2006). Violence between the police and the public: Influences of work-related stress. Journal of Research in Personality. Vol. 33(5), October 2006, Pages 494-497. doi: $10.1177 / 0093854806288039$

McCarty, W., Zhao, J., \& Garland, B. (2007). Occupational stress and burnout between male and female police officers: Are there any gender differences? Policing: An International Journal of Police Strategies and Management, 30(4). 672 - 691. doi.org/10.1108/13639510710833938

Murtagh, C. (2010). Police Stress \& Alcohol Abuse (Doctoral dissertation, Brandeis University). USA. Retrieved from: http://www.worldcat.org/title/police-stress-alcohol-abuse/oclc/666399404

Ostrom, B. \& Job, C. (1986). The Influence of Domestic and International Politics. Journal of Conflict Resolution, Vol. (35), No. 2, 307-332. doi: 10.1177/0022002791035002008

Stinchcomb, J. (2004). Searching for stress in all the wrong places: Combating chronic organizational stress in policing. Police Practice and Research, 5(3), 259-277. doi: 10.1080/156142604200227594

Swanson, C. R., Territo, L., \& Taylor, R. W. (1988). Police administration: Structures, processes, and behavior. Macmillan. NY.

Violanti, J. M. (1993). What Does High Stress Police Training Teach Recruits? An Analysis of Coping. Journal of Criminal Justice, 21, 411-417. doi.org/10.1016/0047-2352(93)90022-F

Violanti, J. M., \& Aron, F. (1995). Police stressors: Variations in perception amongpolice personnel. Journal of Criminal Justice, 23, 287-294. doi.org/10.1016/0047-2352(95)00012-F

Waters, J. \& Ussery, W. (2007). Police stress: history, contributing factors, symptoms, and interventions. Policing: An International Journal of Police Strategies andManagement, 30(2). 1690188.

doi.org/10.1108/13639510710753199 